\title{
Structural Analysis of Polyethylene Fibers by Solid State High Resolution NMR; the Distribution of ${ }^{13} \mathrm{C}$ Spin-Lattice Relaxation Times
}

\author{
Atsushi KaJI, Atsuhiko Yamanaka, and Masao Murano \\ Katata Research Center, Toyobo Co., Ltd., 2-1-1 Katata, \\ Otsu, Shiga 520-02, Japan
}

(Received March 16, 1990)

\begin{abstract}
C}$ solid state high resolution NMR spectra of ultra high-molecular weight polyethylene fibers (UHF) and high-density polyethylene fibers (HDF) were measured at room temperature. The ${ }^{13} \mathrm{C}$ spin-lattice relaxation decay curves for the main lines, assigned to the orthorhombic crystalline phase (ORC), were composed of multiple components and analyzed by non-linear least squares method. Although it was difficult to determine the number of components, the distribution curve of $T_{1 \mathrm{C}} \mathrm{S}$ was obtained and the origin discussed in relation to the crystalline structure of the polyethylene fibers. Three component analysis gave fairly good agreement. The ambiguity of the component analysis is due to the distribution of the $T_{1 \mathrm{C}}$ of each component. The longest $T_{1 \mathrm{C}}$ component had a narrow distribution but the other components were spread over a wide range of $T_{1 \mathrm{C}}$. The $T_{1 \mathrm{C}}$ value of the each component is shorter in HDF than in UHF.
\end{abstract}

KEY WORDS UHMWPE / Solid-State NMR / CP-MAS / Relaxation Time $/ T_{1} /$ Distribution / Phase Structure /

Solid state high resolution NMR is one of the most useful methods for chemical analysis like solution NMR and also can be applied to studies on the physical properties of solid materials. One of the latter applications is based on the spin relaxation behavior which is related to molecular mobility. There have been a lot of studies on the NMR relaxation times in relation to molecular motion. ${ }^{1-6}$ The mode of molecular motion is also studied by deuterium NMR..$^{7-10}$ It is not so easy to interpret the relaxation parameters concerning to the molecular mobility. There are several kinds of relaxation parameters such as spin-lattice relaxation times $\left(T_{1 \mathrm{H}}, T_{1 \mathrm{C}}\right)$, spinlattice relaxation times in the rotating frame $\left(T_{1 \rho \mathrm{H}}, T_{1 \rho \mathrm{C}}\right)$, spin-spin relaxation times $\left(T_{2 \mathrm{H}}\right.$, $T_{2 \mathrm{C}}$ ), and cross-polarization parameters. The relaxation times are related to some correlation times $(\tau)$ by respective correlation functions depending on their relaxation mechanisms.
Restricting to the dipole-dipole relaxation mechanisms, the correlation time $\tau$ changes according to the type of molecular motion. There might be a number of different $\tau$ 's or its distribution in amorphous polymers. ${ }^{11}$ Even though there are a lot of relaxation mechanisms, the equivalent nuclei have single relaxation times in the homogeneous system. Their individual relaxation times can be measured by solid state high resolution NMR because the inequivalent nuclei can be distinguished by the chemical shifts.

In the case of inhomogeneous polymer system such as a blend polymer or semicrystalline polymer like polyethylene, there exist domains having different mobilities such as crystalline and amorphous phase. The morphology of inhomogeneous polymer system has been investigated by ${ }^{1} \mathrm{H}$-spin diffusion. ${ }^{12-15}$ Several $T_{1 \mathrm{C}} \mathrm{s}$ can be observed for different polyethylene samples by solid state high 
resolution NMR. ${ }^{16-22}$ It is necessary to estimate the respective relaxation times before investigating the molecular motion mechanism. Hitherto the longest component was only studied, ${ }^{16,17}$ or the relaxation decay curves were resolved into several components. ${ }^{18-22}$ There should be some distribution of relaxation times in the polymer system, but we cannot find any report investigating the distribution of relaxation times. It would be possible to characterize the inhomogeneity of domains in the polymer by analyzing the distribution.

We studied the phase structure of ultra highmolecular weight polyethylene (UHMWPE) fibers (UHF) by solid state high resolution NMR. ${ }^{23}$ We measured carbon spin-lattice relaxation time $\left(T_{1 \mathrm{c}}\right)$ of the crystalline phase and found that the crystalline signal is composed of several components having different $T_{1 \mathrm{c}}$. It was necessary to postulate the number of components for dividing the relaxation decay curve corresponding to respective ones. However, there were some difficulties to estimate the number of components having different relaxation times, because we had no idea of the number based on morphological nor dynamical model. We try to make this problem clear in this paper and discuss domains in polyethylene fibers.

\section{EXPERIMENTAL}

\section{Materials}

UHMWPE $\left(M_{w} \fallingdotseq 2 \times 10^{6}\right)$ fibers (UHF) were produced by gel drawing according to Smith et al. ${ }^{24}$ The crystallinity of UHF estimated from density was about $90 \%$. The tensile modulus was about $130 \mathrm{GPa}$ and the strength was about $3 \mathrm{GPa}$. HDPE $\left(M_{w} \fallingdotseq 6 \times\right.$ $10^{4}$ ) fibers (HDF) were prepared by melt drawing by the method of Capaccio et al. ${ }^{25}$ The crystallinity of HDF estimated from density was about $65 \%$. The modulus and strength of HDF were about $40 \mathrm{GPa}$ and $1 \mathrm{GPa}$, respectively.
NMR Measurement and Relaxation Time

\section{Analysis}

Solid state high resolution ${ }^{13} \mathrm{C}$ NMR spectra were measured using a Varian XL-300 $\left({ }^{13} \mathrm{C}\right.$; $75.5 \mathrm{MHz}$ ) at ambient temperature. Dipole decoupling power $\left(\gamma B_{1} / 2 \pi\right)$ was about $50 \mathrm{kHz}$ and spin lock power in cross-polarization was about $33 \mathrm{kHz}$. These powers might be insufficient for crystalline polymers like UHF. These points should be in mind for quantitative analysis as well as cross-polarization coefficients. Careful adjustment of the spectrometer was necessary to get sufficiently narrow line width (about $30 \mathrm{~Hz}$ for ORC signal in UHF). The magic angle sample spinning rate was about $3.5 \mathrm{kHz}$.

We measured $T_{1 \mathrm{C}}$ relaxations by Torchia's method. ${ }^{26}$ Each $T_{1 \mathrm{C}}$ decay curve was reconstructed by least squares method in two ways. One is analysis assuming several components and both of their intensities and $T_{1 \mathrm{c}}$ values are variables. In the other analysis, $T_{1 \mathrm{c}}$ values are distributed and only their intensities are varied to get the best fitting. We call these methods for convenience sake as component analysis and distribution analysis, respectively. The calculation method employed here was the simplex method. The convergent rate of this method was not so fast but this method has a lot of merits. The calculation does not diverge. If it fell into a local minimum, it is easy to resume calculation to get better fitting. The influence of initial values on calculation results is small. It is also easy to use a non-negative restriction.

\section{RESULTS AND DISCUSSION}

\section{NMR Spectra and $T_{1 \mathrm{C}}$ Measurements}

Typical CP-MAS spectra of UHF and HDF are shown in Figure 1. Main peaks are assigned to the crystalline phase (orthorhombic crystal; ORC). ${ }^{22,23,27-29}$ A small signal at lower field in UHF is due to monoclinic crystal phase (MCC). ${ }^{22,23,27-29}$ The signal of the noncrystalline phase (NC) is observed in HDF at 


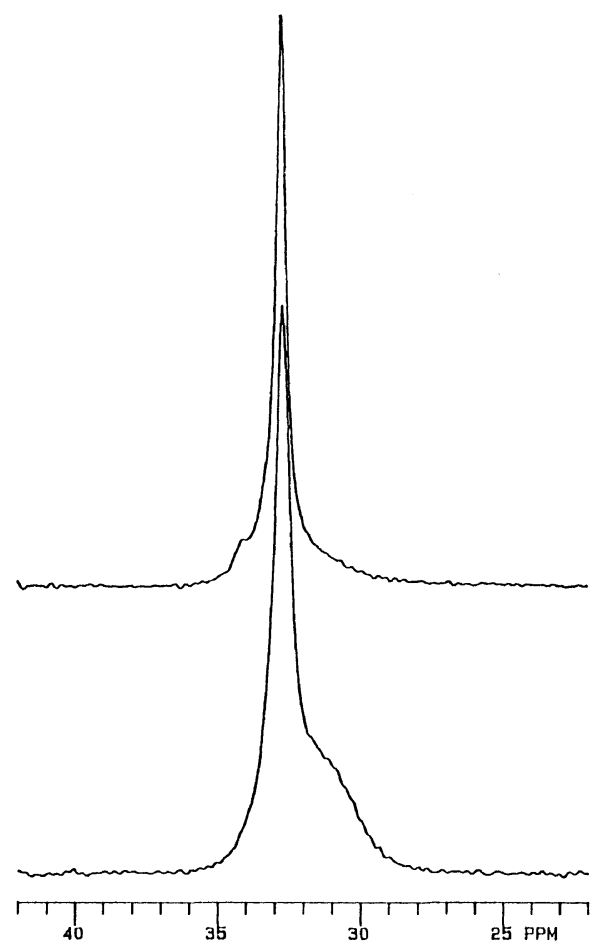

Figure 1. CP-MAS spectra of PE fibers. Upper, UHF; lower, HDF.

higher field. The NC signal in UHF is very small because of its high crystallinity. The NC signal is spread out and not clearly separated from the crystalline signal. The semi-logarithmic plots of $T_{1 \mathrm{C}}$ relaxation decays are shown for the crystalline signals in Figure 2. Each decay indicates that there exist multiple components with different $T_{1 \mathrm{C}} \mathrm{s}$.

\section{Component Analysis}

The tangent line is usually cut from the longer delay time part in the observed decay curve. This tangent line is due to the longest relaxation component. The value of this tangent line is subtracted from the observed line and the result is plotted again. Then we obtain the second tangent line from this plot in the same way as the first one. This procedure is repeated until no further tangent line is obtained. It is difficult, however, to use this method for decay curves containing several

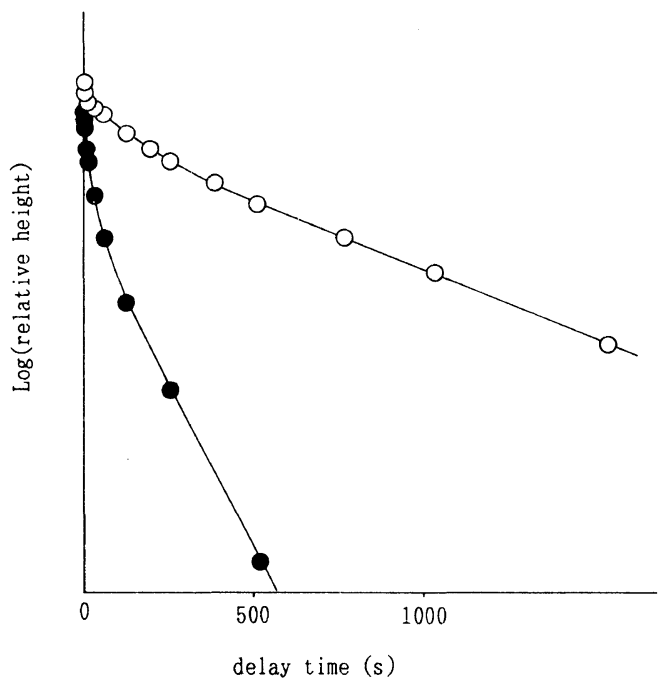

Figure 2. $T_{1 \mathrm{C}}$ decay curves of the crystalline signals. $\bigcirc$, UHF, , HDF.

Table I. $T_{1 \mathrm{C}}$ of the components giving crystalline signals in polyethylene fiber

\begin{tabular}{ccc}
\hline & $T_{1 \mathrm{c}} / \mathrm{s}$ & Ratio/\% \\
\hline \multirow{3}{*}{ UHF } & 1907 & 85 \\
& 114 & 10 \\
& 5.6 & 5 \\
HDF & 449 & 64 \\
& 39 & 26 \\
& 1.6 & 10 \\
\hline
\end{tabular}

components, because the observed line is curved and ambiguity increases in the shorter $T_{1 \mathrm{C}}$ region. The number of components cannot be decided.

When the observed signal consists of several $T_{1 \mathrm{C}}$ components, the relaxation decay in Figure 2 can be represented by eq 1 .

$$
\left\langle X_{\mathrm{o}}\right\rangle_{j}=2\left\{\sum_{i=1}^{n} A_{i} \exp \left(-t / T_{1 \mathrm{C}_{i}}\right)\right\}_{j}
$$

where $\left\langle X_{0}\right\rangle_{j}$; observed signal intensity at data point no. $j, n$; number of components, $A_{i}$; intensity of $i$ th component, $t$; delay time, $T_{1 \mathrm{C}_{i}}$; $T_{1 \mathrm{C}}$ value of $i$ th component.

We get the best set of $A_{i}, T_{1 \mathrm{C}_{i}}$ by non-linear least squares method using eq 1 assuming the 
number " $n$ ". The results obtained assuming $n=3$ for the components giving the crystalline signal are shown in Table $\mathrm{I}^{23}$

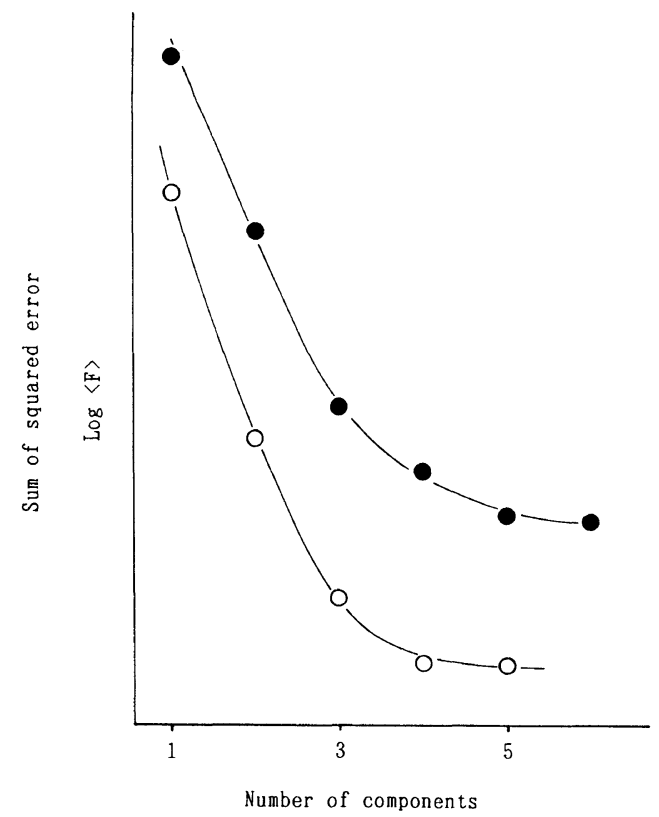

Figure 3. The sum of squared erros $(\langle F\rangle)$ against the number of components assumed. $\mathrm{O}, \mathrm{UHF} ; \mathrm{O}, \mathrm{HDF}$.
The longest $T_{1 \mathrm{C}}$ component reveals longer $T_{1 \mathrm{c}}$ value and greater fraction in UHF than in HDF. Molecules in the crystal of UHF may possibly have less mobility. However, it is difficult to interpret the $T_{1 \mathrm{C}}$ value of the other components because these values depend on the number " $n$ ".

When the number of components can be physically determined, it is excellent to utilize the computer fitting by this method. If not, the physical meaning of the assumed number of components cannot be made clear by fitting.

The sum of the finally achieved squared errors $\langle F\rangle$ is written as eq 2 .

$$
\langle F\rangle=\sum_{j=1}^{m}\left(\left\langle X_{\mathrm{o}}\right\rangle_{j}-\left\langle X_{\mathrm{c}}\right\rangle_{j}\right)^{2}
$$

where $\left\langle X_{0}\right\rangle_{j}$; signal intensity observed at the data point no. $j,\left\langle X_{\mathrm{c}}\right\rangle_{j}$; signal intensity calculated at the data point no. $j, m$; the number of data points.

The plot of $\langle F\rangle$ against the number of components is shown in Figure 3. $\langle F\rangle$ decreases exponentially as the component

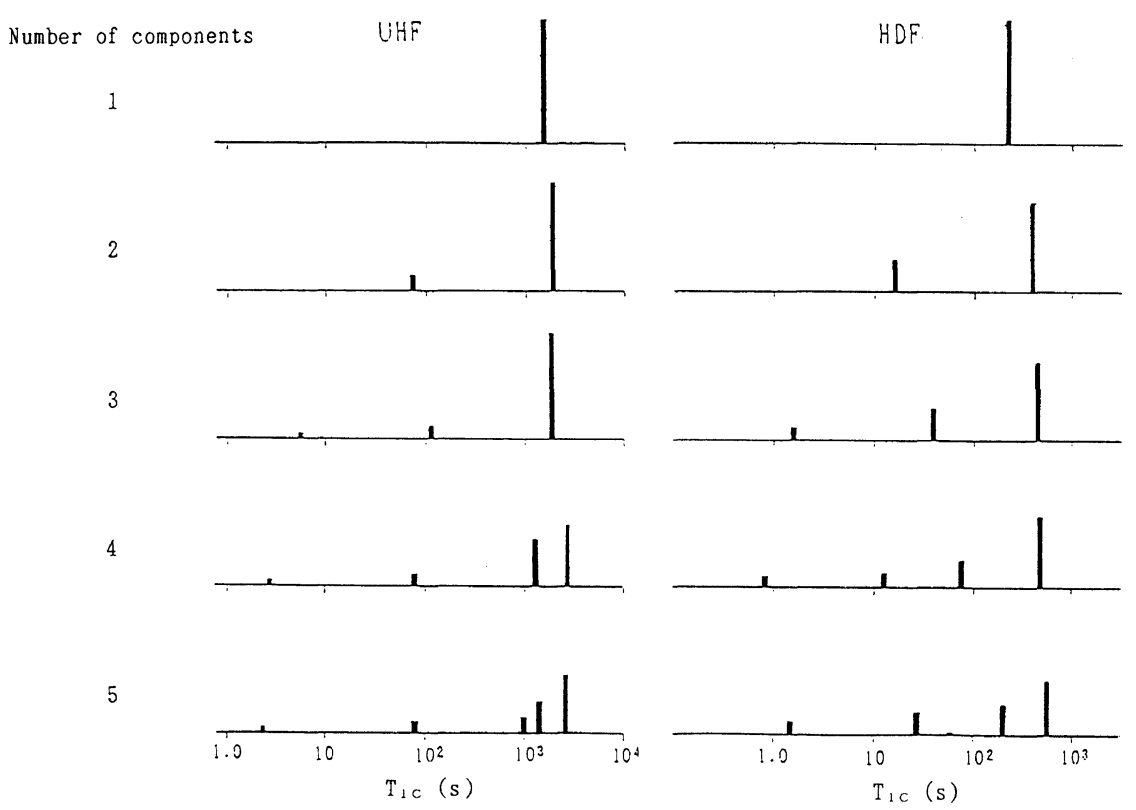

Figure 4. The results of the least squares method. The number of components (" $n$ ") assumed are changed from 1 (top) to 5 (bottom). The horizontal axis is the logarithm of $T_{1 \mathrm{C}}$ and the height of the bar represents relative intensity. 
number increases until 3 (in UHF) or 4 (in HDF). These numbers are the minimum numbers of the components which can reproduce the observed decay curves. However, these numbers do not mean the exact number of components. The calculation has reached the limit of the fluctuation in observed line. The minimum value of $\langle F\rangle$ for UHF is smaller than that for HDF because the fluctuation of observed relaxation decay line is smaller. If we have higher $\mathrm{S} / \mathrm{N}$ ratio data, the smaller $\langle F\rangle$ value can be obtained.

If we change the number of components, both the intensity and $T_{1 \mathrm{C}}$ of each component change as shown in Figure 4. The $T_{1 \mathrm{C}}$ value of the longest $T_{1 \mathrm{C}}$ component changes little in both cases (UHF and HDF). In UHF, when the number of components increases more than 3 , the longest $T_{1 \mathrm{C}}$ component is only subdivided. The substantial number of components and their $T_{1 \mathrm{C}} \mathrm{S}$ are almost unchanged. But the $T_{1 \mathrm{C}}$ value of the shortest $T_{1 \mathrm{C}}$ component is slightly shortened at more than 3 components. The relaxation decay of UHF can be reconstructed substantially by 3 components. In the case of $\mathrm{HDF}$, the longest $T_{1 \mathrm{C}}$ component is unchanged but the other components are split into several components. It is difficult to determine the number and $T_{1 \mathrm{C}}$ values of the components in HDF.

\section{Distribution Curve Analysis}

We can get the distribution curve of $T_{1 \mathrm{C}}$ values from eq 1 . The $T_{1 \mathrm{C}}$ values are predetermined and spread over the time range and fixed. In this paper, $T_{1 \mathrm{C}}$ were selected in geometrical progression since the data points of the $T_{1 \mathrm{C}}$ decay curves had been selected in the same manner. Then minimization of squared errors is made by varying the intensities of the components only. More number components are needed to achieve the equal $\langle F\rangle$ value than in the component analysis in which both the intensities and $T_{1 \mathrm{C}}$ can be varied. In this method, $\langle F\rangle$ also decreases with increasing " $n$ " and reaches a minimum for

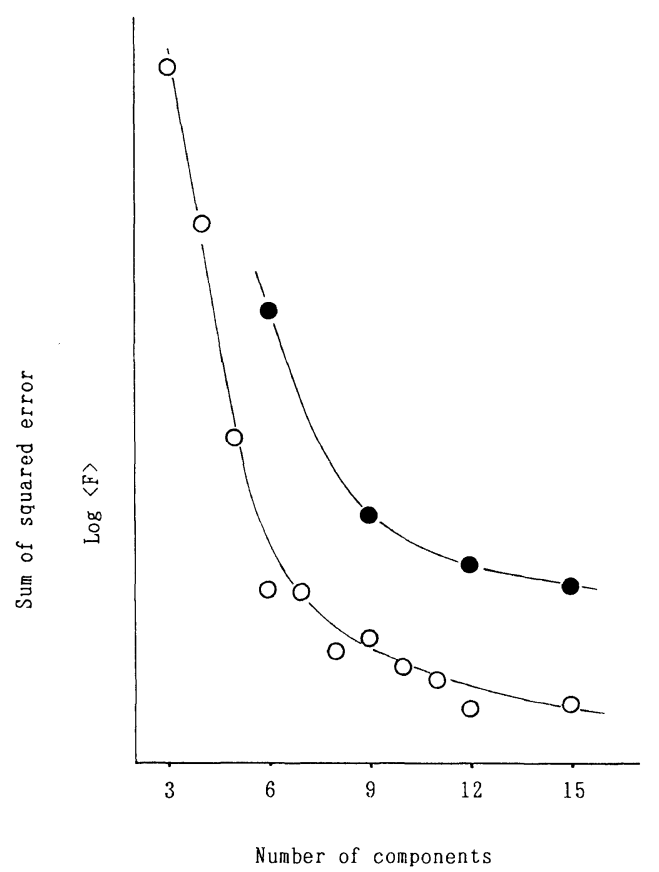

Figure 5. The sum of squared errors $(\langle F\rangle)$ against the number of points used. $\mathrm{O}, \mathrm{UHF} ; \mathrm{O}, \mathrm{HDF}$.

$n>12$ as shown in Figure 5. The distributions of $T_{1 \mathrm{C}} \mathrm{s}$ thus obtained are shown in Figure 6. The results of the 3 component analysis are also shown as histograms in this figure. A comparison of the results on the component analysis with those on the distribution analysis indicates the origin of the ambiguity in the component analysis. There are principally trimodal distributions for the crystalline signals. The longest $T_{1 \mathrm{C}}$ fraction should be assigned to the crystalline component and the other two fractions may be assigned to intermediate regions. The average values of the longest $T_{1 \mathrm{C}}$ fractions are in fairly good agreement with the $T_{1 \mathrm{C}}$ values of longest $T_{1 \mathrm{C}}$ components in both UHF and HDF, though the other components are a little different. They are distributed over a wide range of $T_{1 \mathrm{C}}$. In the case of UHF, the distribution of the longest $T_{1 \mathrm{C}}$ fraction, which seems to be rather narrow, should correspond to the distribution of the size of crystals. The shortest one of the 3 components corresponds 


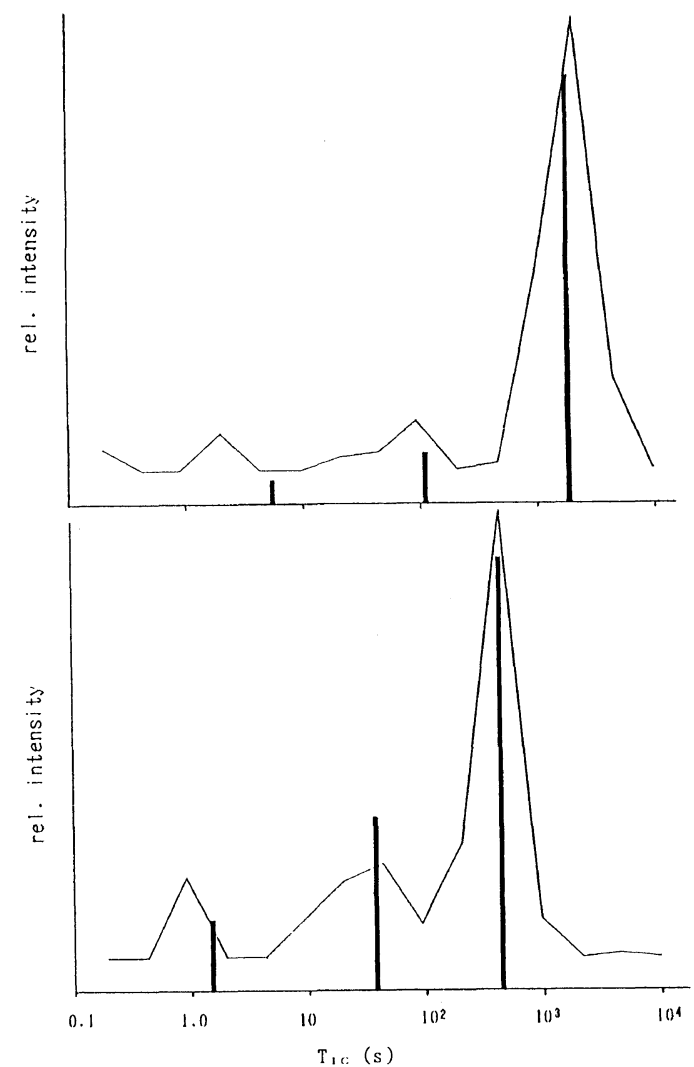

Figure 6. The distribution of $T_{1 \mathrm{C}} \mathrm{S}$ estimated from the crystalline signal with the results of three component analysis (histograms). Upper, UHF; lower, HDF. The horizontal axis is the logarithm of $T_{1 \mathrm{c}}$ and the vertical axis is relative intensity.

to a mean of $T_{1 \mathrm{C}} \mathrm{s}$ from about 0.2 to $20 \mathrm{~s}$. There is a small hump at about $2 \mathrm{~s}$, in fact. These wide spreadings are the cause of the ambiguity of component analysis.

When the $T_{1 \mathrm{C}}$ distribution is separated into three divisions, the average $T_{1 \mathrm{C}}$ value of each is shorter in HDF than in UHF. Since the ${ }^{13} \mathrm{C}$ spin diffusion effect may be significant for the components with $T_{1 \mathrm{C}} \mathrm{s}$ more than a hundred seconds and the $T_{1 \mathrm{C}}$ values are assumed to depend on the crystal size, the crystal size is smaller in HDF than in UHF. The $T_{1 \mathrm{C}}$ values of the other divisions should be explained by molecular mobility which is more significant than the spin diffusion effect in shorter $T_{1 \mathrm{C}}$.

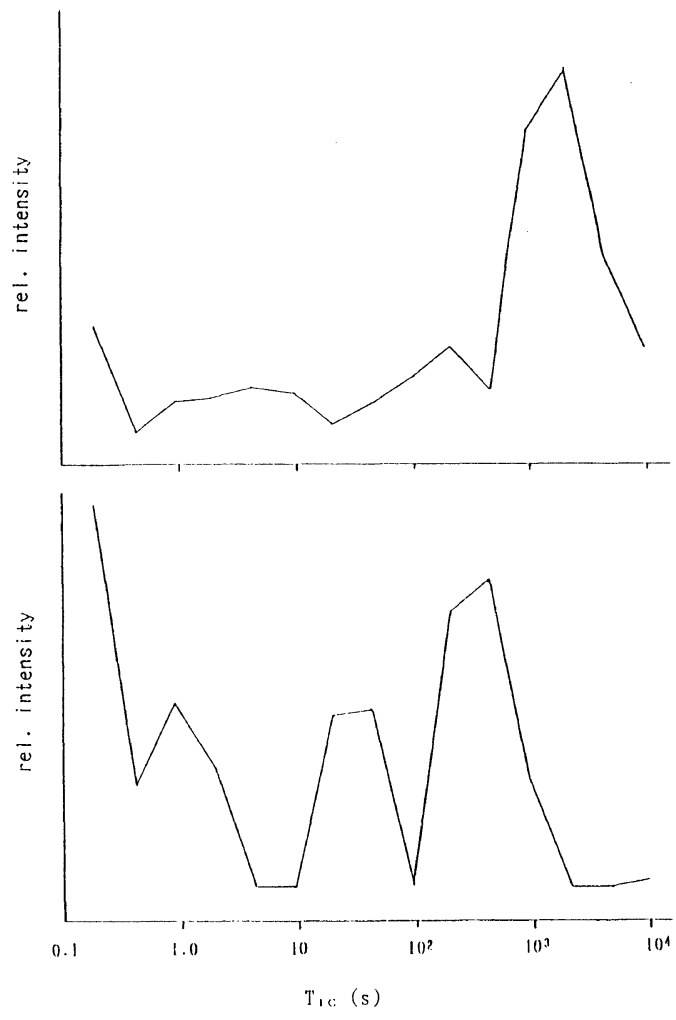

Figure 7. The distribution of $T_{1 \mathrm{c}} \mathrm{S}$ estimated from integral intensity. Upper, UHF; lower, HDF. The horizontal axis is the logarithm of $T_{1 \mathrm{C}}$ and the vertical axis is relative intensity.

The mobility of the molecules even in morphologically similar region may be higher in HDF than in UHF.

The $T_{1 \mathrm{C}}$ analysis is so far applied to components giving the crystalline signal. We also obtained $T_{1 \mathrm{C}}$ distribution curves for the integrated intensity decay curves including the NC signal. The results are shown in Figure 7. They are similar to the results from the crystalline signals at longer $T_{1 \mathrm{C}}$ region. But because of the signal width and contribution of the NC signal, the relative intensity of the shorter $T_{1 \mathrm{C}}$ region is larger in this analysis. Since the $T_{1 \mathrm{C}}$ of $\mathrm{NC}$ does not exceed $1 \mathrm{~s}$, the $T_{1 \mathrm{C}}$ distribution of longer than $1 \mathrm{~s}$ is due to components which give crystalline signal. There can be seen four components (NC, 
crystalline, two types of intermediate region) principally. One of the two intermediate region might be the borders on $\mathrm{NC}$ and the other one on the crystal. In HDF, these four components are observed distinctively. They may be distinguished by the molecular motion mode. It might be considered that the molecules in the crystals can vibrate only, the ones in longer $T_{1 \mathrm{C}}$ components of the intermediate region can flip, the ones in shorter $T_{1 \mathrm{C}}$ components of the intermediate region can rotate along the chain and the ones in $\mathrm{NC}$ can move randomly. The amounts of shorter $T_{1 \mathrm{C}}$ components are smaller in UHF than in HDF. This may mean that not only the NC but also the intermediate regions are smaller in UHF than in HDF. The shorter $T_{1 \mathrm{C}}$ components are distributed over a wider range in UHF than in HDF. Because of the high molecular weight and ultra drawing in UHF, small $\mathrm{NC}$ and intermediate regions consist of the molecules tied between crystals mainly. The molecular motion is restricted by each crystal and then some $T_{1 \mathrm{C}} \mathrm{s}$ may shift to the longer region depending on the restriction.

\section{CONCLUSIONS}

1. Analysis of the distribution of the $T_{1 \mathrm{C}}$ values is useful in investigating the nonhomogeneity of polyethylene fibers.

2. The structures of crystalline and intermediate regions were investigated by this method. The region giving the crystalline signal mainly consists of three components. They should be crystals and two types of intermediate regions. Since each of them has a distribution, analysis by assuming the number of components has ambiguity.

3. UHF is not only crystal rich and NC poor, but the intermediate regions are small. Though $T_{1 \mathrm{C}} \mathrm{s}$ of the intermediate regions are spread over a wide range of $T_{1 \mathrm{C}}$, they shift a little longer side in UHF than in HDF. It may mean that the molecular motions in those regions of UHF are restricted and the strain cannot be released because the intermediate regions consist of the tied molecules among the crystals.

4. The distribution analysis of the relaxation times as shown in this paper by the $T_{1 \mathrm{C}}$ values will be useful in investigating the non-homogeneity of materials.

\section{REFERENCES}

1. D. E. Woessner, J. Chem. Phys., 36, 1 (1962).

2. F. Heatley and A. Begum, Polymer, 17, 399 (1976).

3. A. Tsutsumi, Molec. Phys., 37, 111 (1979).

4. R. D. de la Batie, F. Laupretre, and L. Monnerie, Macromolecules, 22, 122 (1989).

5. I. Bahar and B. Erman, Macromolecules, 22, 2396 (1989).

6. M. G. Brereton, Macromolecules, 22, 3667 (1989).

7. D. Hentschel, H. Sillescu and H. W. Spiess, Macromolecules, 14, 1607 (1981).

8. J. J. Dumais, L. W. Jerinski, L. M. Leung, I. Gancarz, A. Galambos, and J. T. Koberstein, Macromolecules, 18, 119 (1985).

9. A. Kintanar, K. W. Jerinski, I. Gancarz, and J. T. Koberstein, Macromolecules, 19, 1876 (1986).

10. J. J. Dumais, A. L. Cholli, L. W. Jerinski, J. L. Hedrick, and J. E. McGrath, Macromolecules, 19, 1884 (1986).

11. B. Gabrys, F. Horii, and R. Kitamaru, Macromolecules, 20, 175 (1987).

12. R. A. Assink, Macromolecules, 11, 1233 (1978).

13. T. T. P. Cheung and B. C. Gerstein, J. Appl. Phys., 52, 5517 (1981).

14. P. Caravatti, P. Neuenschwander, and R. R. Ernst, Macromoleculs, 18, 119 (1985).

15. P. Caravatti, P. Neuenschwander, and R. R. Ernst, Macromolecules, 19, 1889 (1986).

16. D. L. Axelson, J. Polym. Sci., Polym. Phys. Ed., 20, 1427 (1982).

17. D. L. Axelson, L. Mandelkin, R. Popli, and P. Mathieu, J. Polym. Sci., Polym. Phys. Ed., 21, 2319 (1983).

18. B. Schroter and A. Posern, Makromol. Chem., 182, 675 (1981).

19. B. Schroter and A. Posern, Makromol. Chem. Rapid Commun., 3, 623 (1982).

20. M. Ito, H. Serizawa, K. Tanaka, W. P. Leung, and C. L. Choy, J. Polym. Sci., Polym. Phys. Ed., 21, 2299 (1983).

21. F. Horii, R. Kitamaru, S. Maeda, A. Saika, and T. Terao, Polym. Bull., 13, 179 (1985).

22. R. Kitamaru, F. Horii, and K. Murayama, Macromolecules, 19, 636 (1986).

23. A. Kaji, Y. Ohta, H. Yasuda, and M. Murano, Polym. 
J., 22, 455 (1990).

24. P. Smith and P. J. Lemstra, US Patent, 4,344,908 (1982).

25. G. Capaccio, F. Smith, and I. M. Ward, Japan Patent, 50-126921 (1975).

26. D. A. Torchia, J. Magn. Reson., 30, 613 (1978).
27. D. L. VanderHart and F. Khoury, Polymer, 25, 1589 (1984).

28. M. Takenaka, T. Yamanobe, T. Komoto, I. Ando, and H. Sato, Solid State Commun., 61, 563 (1987).

29. T. Yamanobe, T. Sorita, T. Komoto, I. Ando, and H. Sato, J. Mol. Structure, 131, 267 (1985). 